\title{
Spectroscopic diagnostic for the mineralogy of large dust grains
}

\author{
M. Min, C. Dominik, and L. B. F. M. Waters \\ Astronomical institute Anton Pannekoek, University of Amsterdam, Kruislaan 403, 1098 SJ Amsterdam, The Netherlands \\ Received 31 October 2003 / Accepted 27 November 2003

\begin{abstract}
We examine the thermal infrared spectra of large dust grains composed of amorphous and crystalline olivine. Strong resonances in the refractive indices result in detectable spectral structure even when the grain is much larger than the wavelength at which it radiates. We apply this to the thermal infrared spectra of compact amorphous and crystalline silicates. The weak resonances of amorphous silicates at 9.7 and $18 \mu \mathrm{m}$ virtually disappear for grains larger than about $10 \mu \mathrm{m}$. In contrast, the strong resonances of crystalline silicates produce emission dips in the infrared spectra of large grains; these emission dips are shifted in wavelength compared to the emission peaks commonly seen in small crystalline silicate grains. We discuss the effect of a fluffy or compact grain structure on the infrared emission spectra of large grains, and apply our results to the dust shell surrounding Vega.
\end{abstract}

\section{Introduction}

Infrared spectroscopy is an invaluable tool for the study of the structure and composition of interstellar and circumstellar dust. In the infrared, functional groups in a dust grain lead to spectral features in the absorption cross section of a grain. These features can be detected in absorption against a strong infrared background source, or as emission features if the grains are warm enough to emit thermally in this wavelength region. The precise shapes and positions of such features contain information about grain size, shape and detailed chemical composition (Bohren \& Huffman 1983).

It is generally assumed that dust grains need to be small in order to produce spectral structure. When grains are large, each individual grain becomes optically thick at infrared wavelength, and the features in the absorption cross section are expected to weaken with increasing grain size and eventually to disappear completely. In most environments in interstellar space, small dust grains dominate the overall grain surface available and therefore also dominate the total absorption cross section. There are, however, environments where small grains are heavily depleted. In particular in gas-poor circumstellar disks, small dust grains are removed by radiation pressure and larger grains start to dominate the interaction with radiation. For example, the zodiacal dust in the solar system is dominated by large grains since grains smaller than about 1 micron are quickly lost (Burns et al. 1979). This effect becomes even stronger in debris disks around A-type main sequence stars like Vega, where the increased luminosity shifts the typical blowout size to about 10 micron (Artymowicz 1988). Even stronger effects may exist in disks around (post)-AGB stars, where already grains of $\mathrm{mm}$ size will be lost unless the disk is optically thick and gas-rich (Dominik et al. 2003).

Send offprint requests to: M. Min, e-mail: mmin@science.uva.nl
We therefore examine in this paper the optical properties of large dust grains. In Sect. 2 we show that the strong resonances observed in crystalline materials lead to observable structure in the infrared spectra of large dust grains. In Sect. 3 we apply these results to the dust shell around Vega. In Sects. 4 and 5 we discuss and summarize our results.

\section{Infrared emission spectra}

In order to calculate the thermal emission spectrum of a dust shell we need to know the emission cross sections of the dust grains. With grain size, shape and chemical composition known, the emission cross section per unit mass as a function of wavelength can be calculated from the wavelength dependent bulk refractive index. In grains much smaller than the wavelength of radiation, a resonance in the bulk refractive index causes a so-called surface mode (Bohren \& Huffman 1983). These surface modes are strong enhancements of the absorption cross section and are detected as emission features in the thermal emission spectra of these grains. For larger grains, the contrast in the absorption features is diminished and it is generally assumed that the thermal emission from large particles will be a smooth blackbody without a characteristic signature of the mineralogy. However, this assumption only holds if the refractive index is close to unity over the entire wavelength range. For the resonances in crystalline silicates, both the real and imaginary part of the refractive index can reach values up to $\sim 9$. For large particles with these values of the refractive index, most radiation will be reflected off the surface of the particle and will not penetrate the grain. This leads to a significant reduction of the grain absorption efficiency at these wavelengths. Therefore, we also expect to find a corresponding decrease of the emission efficiency, at wavelengths where there is a resonance in the bulk refractive index (Bohren \& Huffman 1983). This is illustrated 


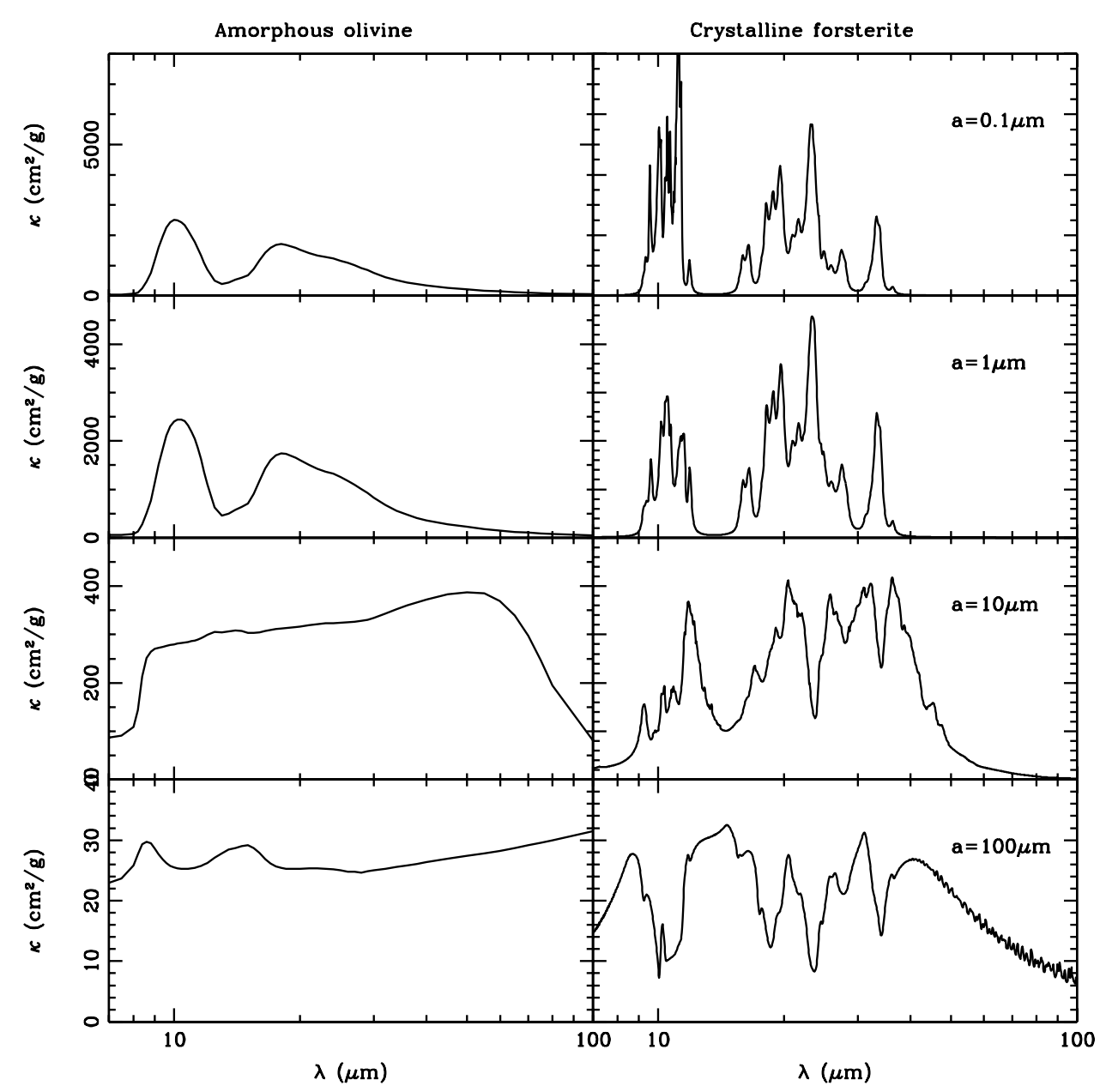

Fig. 1. The emission cross sections per unit mass, $\kappa$, for various sizes of amorphous olivine grains (left panels) and crystalline forsterite grains (right panels) as functions of the wavelength. The emissivities were calculated using a uniform distribution of spheroids (UDS). The refractive index data for the crystalline forsterite are taken from Servoin \& Piriou (1973), for the amorphous olivine the data are taken from Dorschner et al. (1995). Note the different $y$-scales for the different grain sizes.

in Fig. 1 where the emission cross sections per unit mass are plotted for grains composed of amorphous olivine and crystalline forsterite for different grain sizes. Moving from small to large grains, the structure in the spectrum of amorphous olivine virtually disappears, to a level which makes detection in astrophysical environments extremely hard. However, as pointed out earlier by Lynch \& Mazuk (2000), the spectral structure never disappears completely. This effect becomes much more prominent for the crystalline forsterite: the features in this spectrum change from emission peaks to emission dips. The remaining contrast even for very large grains is strong enough to be detected in astrophysical sources. Generally the features at shorter wavelengths and the strongest features are the first to disappear and change into emission dips. Most dips in the spectra of the large grains are slightly red-shifted compared to the corresponding emission enhancements in the small grain spectra. When the grain size is increased even more, the overall shape of the spectrum will flatten but the spectral structure remains visible at about the same contrast level.

In order to calculate the emission cross sections, a particle shape has to be assumed. We used a uniform distribution of spheroids which is in very good agreement with laboratory measurements of the mass absorption coefficients of small crystalline silicate grains (Min et al. 2003b). The cross sections were calculated using the method described in Min et al. (2003a). For the large particles, the emission efficiencies are the same for all convex shaped particles. Calculations for homogeneous spheres using Mie theory (Mie 1908) will therefore give the same emission spectrum in the large particle limit.

It is important to note that for large compact grains the emission cross section per unit mass is very low compared to small grains - this can be seen from the different scales used in Fig. 1. Also, the contrast of the emission dips created in large grains is weaker than the emission peaks due to small grains. In most spectra, the emission by small grains, which is much more efficient, will be dominating. The spectral signature of the large compact dust grains should therefore be observable only in environments where small grains are heavily depleted.

\section{The spectrum of Vega}

An efficient mechanism to remove small grains from a population of grains is radiation pressure on grains in orbit around a 


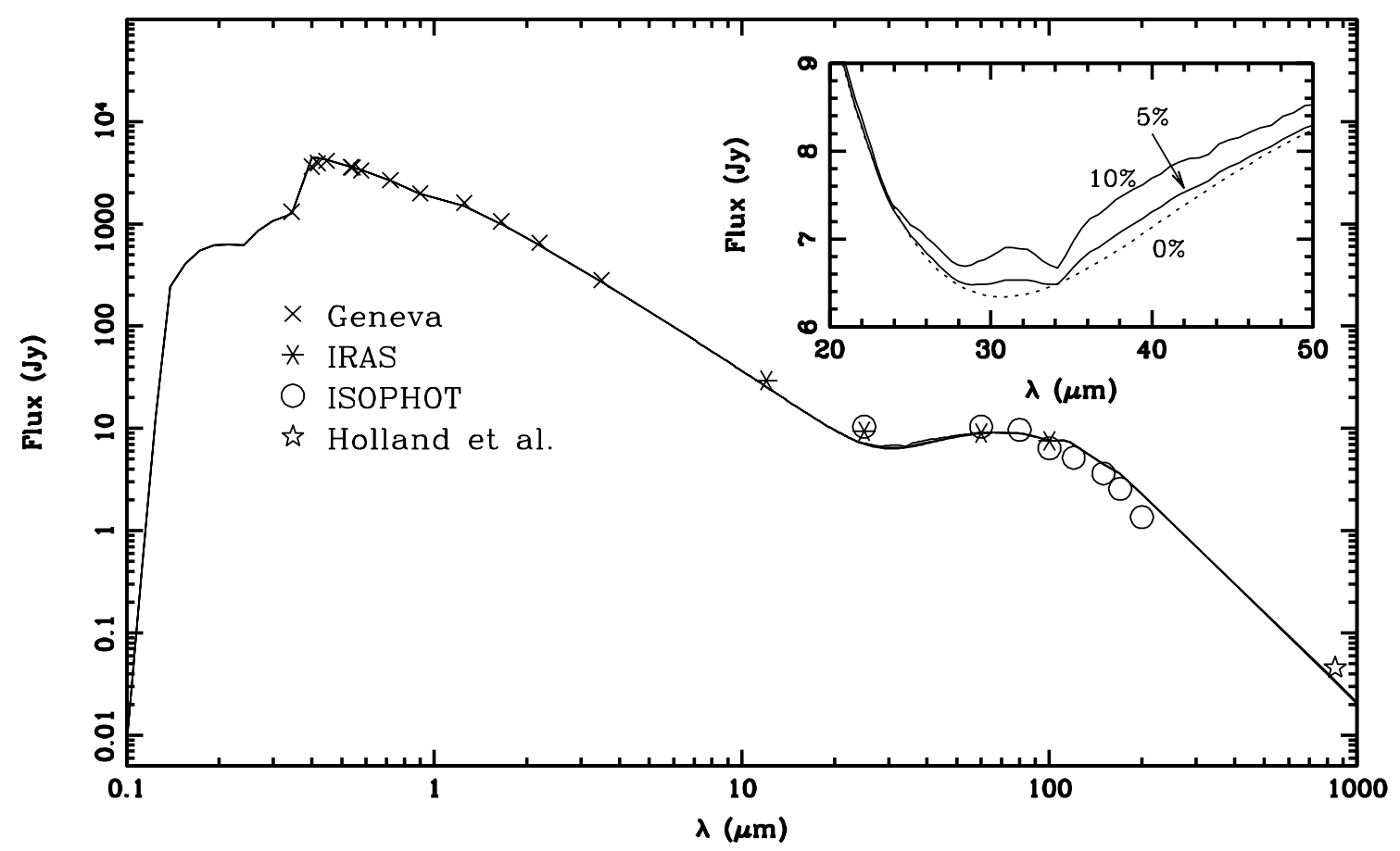

Fig. 2. Model infrared spectra for Vega. The parameters for the model are given in the text. The IRAS data are taken from Walker \& Wolstencroft (1988), the ISOPHOT data are from Heinrichsen et al. (1998) and the submm flux is taken from Holland et al. (1998). The labels with the curves in the inset are the abundances of forsterite for the different models.

star. Due to the efficient absorption, the radiative acceleration on small grains will easily exceed the gravitational force exerted by the stars. If the grains are orbiting in a gas-poor, optically thin circumstellar shell, they will be removed on a Kepler timescale. Such conditions are given in the debris disks around main-sequence stars.

To demonstrate the effects of large compact crystalline grains, we calculated the spectrum of Vega using a dust shell in a distance range of 80-120 AU from the star. The stellar spectrum is represented by a Kurucz model (Kurucz 1993) with $T_{\text {eff }}=9500 \mathrm{~K}$ and $\log g=4$. For the size distribution of the dust grains we used a powerlaw with sizes between 15 and $100 \mu \mathrm{m}$ and a powerlaw index of -3.5 , typical for a collisional cascade (Tanaka et al. 1996). From the collisional model of Dominik $\&$ Decin (2003) and the calculated radiation pressure we estimated the fraction of small particles to be $\$ 0.1 \%$ of the total mass. Calculations show that this mass fraction does not produce significant emission to be visible in the spectrum. The dust is assumed to be a mixture of $20 \%$ amorphous carbon and $80 \%$ olivine by mass. We calculated various models with different mass fraction of crystalline and amorphous olivine. The dust grains are assumed to be compact particles. Since shape effects are negligible for dust grains of these sizes, the optical properties are calculated using homogeneous spheres. The refractive index data for the amorphous carbon are taken from Preibisch et al. (1993). The dust temperatures are computed self-consistently from the local radiation field. Typical values for the dust temperatures found in this way are $\sim 80 \mathrm{~K}$. The total dust mass used in order to fit the photometry points is $\sim 1 / 10$ of a lunar mass which is in agreement with the estimate by Dent et al. (2000).
The model spectrum is shown in Fig. 2, which runs reasonably well through the observed photometry points. It should be stressed here that the presented spectrum is an example to demonstrate the effect of large grains. We do not claim to present the best fit to the available data points. The inset in Fig. 2 shows an enlargement of the 20 to $50 \mu \mathrm{m}$ region. A prominent dip in the spectrum at $34 \mu \mathrm{m}$ is caused by large compact forsterite grains. Even with only $5 \%$ of the mass present as compact forsterite grains, the dip would most likely be detectable by SIRTF.

\section{Discussion}

\subsection{Grain composition}

In Sect. 3 we have shown that a fraction of about $5 \%$ in compact forsterite grains would produce a detectable feature in the spectrum of Vega. It is important to note that this mass fraction should be present as seperate homogeneous grains. If the forsterite material is present as small inclusions inside an amorphous matrix, a significantly larger fraction of forsterite would be required to produce a spectral signature.

\subsection{Grain structure}

The structure of the dust grains is an important parameter that contains information about its formation and processing history. As a first rough classification we can distinguish between fluffy and compact particles. The structure of a grain can be traced back to its formation process. Dust grains that are grown by coagulation of small (submicron sized) grains will show a very fluffy open structure (Blum et al. 2000), similar to the 
structure observed in interplanetary dust particles (IDP). Such grains are also expected to result from the destruction of primitive planetary bodies such as small cometary nuclei. Compact (non-fluffy) large grains will result from the destruction of larger parent bodies which have been molten and possibly differentiated during the formation process, like large asteroids.

Measurements of the infrared transmission spectrum of a large $(a \approx 20 \mu \mathrm{m})$ fluffy IDP by Molster et al. (2003) still shows the resonances characteristic for submicron grains. This indicates that the infrared spectrum of fluffy particles will still be dominated by the spectral signature of the constituents of the aggregate. In other words, we expect to see the small particle crystalline silicate emission features in the emission spectra of these dust grains. For large compact particles we expect to see a spectral signature as shown for $100 \mu \mathrm{m}$ sized particles in Fig. 1.

\section{Conclusions}

We have developed a new diagnostic tool for determining the mineralogy of large compact dust grains. The thermal emission spectra of large compact crystalline silicate dust grains exhibit characteristic features that can be detected with high signal to noise spectroscopy. These features are emission dips at positions that can be slightly red shifted from the corresponding emission peaks observed for small grains. As an example, a model calculation of the infrared emission from the dust shell around Vega shows that these features should be detectable if the dust grains are compact and contain at least 5\% crystalline forsterite in a seperate grain component. Since there is a clear spectroscopic difference between fluffy and compact dust grains, the spectral signature of crystalline silicates can be used to obtain information on the structure, mineralogy and formation history of the dust grains. Another application of this diagnostic can be the zodiacal dust.

Our model calculations show that if large compact crystalline silicates are present even with modest abundances, this should be detectable as a depression near $34 \mu \mathrm{m}$. If the grains are fluffy, an emission band should be present at a slightly shorter wavelength. Such spectral structure may be measured using the IRS spectrograph on board of SIRTF.

Acknowledgements. We would like to thank J. W. Hovenier for valuable discussions. Comments on an earlier version of this manuscript by F. J. Molster are gratefully acknowledged.

\section{References}

Artymowicz, P. 1988, ApJ, 335, L79

Blum, J., Wurm, G., Kempf, S., et al. 2000, Phys. Rev. Lett., 85, 2426

Bohren, C. F., \& Huffman, D. R. 1983, Absorption and Scattering of Light by Small Particles (New York: Wiley)

Burns, J. A., Lamy, P. L., \& Soter, S. 1979, Icarus, 40, 1

Dent, W. R. F., Walker, H. J., Holland, W. S., \& Greaves, J. S. 2000, MNRAS, 314, 702

Dominik, C., \& Decin, G. 2003, ApJ, 598, 626

Dominik, C., Dullemond, C. P., Cami, J., \& van Winckel, H. 2003, A\&A, 397, 595

Dorschner, J., Begemann, B., Henning, T., Jäger, C., \& Mutschke, H. 1995, A\&A, 300, 503

Heinrichsen, I., Walker, H. J., \& Klaas, U. 1998, MNRAS, 293, L78

Holland, W. S., Greaves, J. S., Zuckerman, B., et al. 1998, Nature, 392, 788

Kurucz, R. 1993, Model Atmospheres, CD-ROM Nos. 1-18 (Cambridge, Mass.: Smithsonian Astrophysical Observatory)

Lynch, D. K., \& Mazuk, S. 2000, in Thermal emission spectroscopy and analysis of dust, disks, and regoliths, ed. M. L. Sitko, A. L. Sprague, \& D. K. Lynch, ASP Conf. Ser., 196, 127

Mie, G. 1908, Ann. Phys., 25, 377

Min, M., Hovenier, J. W., \& de Koter, A. 2003a, J. Quant. Spec. Radiat. Transf., 79-80, 939

Min, M., Hovenier, J. W., \& de Koter, A. 2003b, A\&A, 404, 35

Molster, F. J., Demyk, A., D'Hendecourt, L., \& Bradley, J. P. 2003, 34th Annual Lunar and Planetary Science Conference, abstract No. 1148

Preibisch, T., Ossenkopf, V., Yorke, H. W., \& Henning, T. 1993, A\&A, 279,577

Servoin, J. L., \& Piriou, B. 1973, Phys. Stat. Sol. (b), 55, 677

Tanaka, H., Inaba, S., \& Nakazawa, K. 1996, Icarus, 123, 450

Walker, H. J., \& Wolstencroft, R. D. 1988, PASP, 100, 1509 